\title{
The Genetic Architecture of Emerging Fungicide Resistance in Populations of a Global Wheat Pathogen
}

\author{
Danilo Pereira ${ }^{1,3}$, Bruce A. McDonald $\mathbb{D}^{1}$, and Daniel Croll $\mathbb{D}^{2, *}$ \\ ${ }^{1}$ Plant Pathology, Institute of Integrative Biology, ETH Zürich, Zürich, Switzerland \\ ${ }^{2}$ Laboratory of Evolutionary Genetics, Institute of Biology, University of Neuchâtel, Neuchâtel, Switzerland \\ ${ }^{3}$ Present address: Max Planck Institute for Evolutionary Biology, August-Thienemann-Str. 2, D-24306 Plön, Germany. \\ *Corresponding author: E-mail: daniel.croll@unine.ch.
}

Accepted: 22 September 2020

Data deposition: This project has been deposited at the NCBI Sequence Read Archive under the BioProject PRJNA606320.

\begin{abstract}
Containing fungal diseases often depends on the application of fungicidal compounds. Fungicides can rapidly lose effectiveness due to the rise of resistant individuals in populations. However, the lack of knowledge about resistance mutations beyond known target genes challenges investigations into pathways to resistance. We used whole-genome sequencing data and association mapping to reveal the multilocus genetic architecture of fungicide resistance in a global panel of 159 isolates of Parastagonospora nodorum, an important fungal pathogen of wheat. We found significant differences in azole resistance among global field populations. The populations evolved distinctive combinations of resistance alleles which can interact when co-occurring in the same genetic background. We identified 34 significantly associated single nucleotide polymorphisms located in close proximity to genes associated with fungicide resistance in other fungi, including a major facilitator superfamily transporter. Using fungal colony growth rates and melanin production at different temperatures as fitness proxies, we found no evidence that resistance was constrained by genetic trade-offs. Our study demonstrates how genome-wide association studies of a global collection of pathogen strains can recapitulate the emergence of fungicide resistance. The distinct complement of resistance mutations found among populations illustrates how the evolutionary trajectory of fungicide adaptation can be complex and challenging to predict.
\end{abstract}

Key words: genome-wide association mapping, evolutionary genomics, trade-offs, pathogenic fungi.

\section{Significance}

Agricultural food production is threatened by the emergence of pathogens with resistance to chemical agents that are often the sole option to limit damage. We combined genome sequencing and experimental work on a global collection of a fungal wheat pathogen to show how the species gained resistance to a major chemical agent. Exploiting constraints associated with resistance could help to make crop production more sustainable.

\section{Introduction}

Fungal pathogens threaten global food security and human health (Fisher et al. 2012), causing economic losses and impacting global poverty (Strange and Scott 2005). Treatment of both animal and plant fungal infections rely on the application of fungicidal compounds that increasingly exhibit a decrease in effectiveness (Fisher et al. 2018).
The emergence of resistance in fungal populations affects nearly all major fungicide groups (Stehmann and de Waard 1996; Sierotzki et al. 2000; Avenot and Michailides 2007). The loss in efficacy is due mainly to the intense selective pressure imposed by continuous fungicide applications based on single active compounds (van den Bosch and Gilligan 2008; Hobbelen et al. 2014). Mutations reducing

(C) The Author(s) 2020. Published by Oxford University Press on behalf of the Society for Molecular Biology and Evolution.

This is an Open Access article distributed under the terms of the Creative Commons Attribution License (http://creativecommons.org/licenses/by/4.0/), which permits unrestricted reuse, distribution, and reproduction in any medium, provided the original work is properly cited. 
fungicide sensitivity are strongly favored by selection and quickly increase in frequency in selected populations (McDonald and Linde 2002; van den Bosch and Gilligan 2008; van den Bosch et al. 2011). The genetic architecture associated with resistant phenotypes will arise from a complex array of mutations and their interactions, in turn affected by the pathogen population biology and characteristics of the fungicide. The mode of action (such as impairing mitochondrial respiration, Yang et al. 2011) and the number of target sites (single- vs. multisite fungicides) will play key roles in defining routes to resistance. Genetic trade-offs impacting fitness (Mikaberidze and McDonald 2015), innate resistance and epistatic effects will also significantly shape the evolutionary process of resistance emergence (Lucas et al. 2015). Hence, deciphering the genetic architecture of emerging fungicide resistance can provide useful insights and potentially identify key factors governing the evolutionary responses of pathogens.

Fungicides from the family of demethylation inhibitors (DMls) are the most widely used molecules in agriculture and human medicine (Fisher et al. 2018). The mode of action is to hinder the biosynthesis of ergosterol through inhibition of the $14 \alpha$-demethylase (CYP51) enzyme, negatively impacting the fungal cell membrane integrity and permeability (Georgopapadakou and Walsh 1996; Lass-Flörl 2011). In this group of fungicides, resistance emerges through different mechanisms, including 1) amino acid mutations in the target protein, 2) overexpression of the gene encoding the target protein, and 3) enhanced transporter activity reducing intracellular concentrations of the fungicide (Becher and Wirsel 2012; Cools and Fraaije 2013). Importantly, resistance in populations may be based on multiple mechanisms and is likely to be constrained by fitness costs (Zhan and McDonald 2013; Mikaberidze and McDonald 2015). Resistance can also emerge multiple times independently within species (Torriani et al. 2009). Structural changes in the CYP51 protein are considered the most common mechanism leading to resistance across species (Deng et al. 2007; Lucas et al. 2015). Highly resistant genotypes can acquire dozens of different mutations in the CYP51 gene in a stepwise manner (Cools and Fraaije 2013). The consequences of the stepwise accumulation of mutations are complex interactions with the genetic background and selection for compensatory mutations (Cools et al. 2010; Lucas et al. 2015; Mullis et al. 2018). Alternative mechanisms to point mutations include copynumber variation of CYP51 paralogs that are frequent in Ascomycota fungi (Deng et al. 2007; Liu et al. 2011; Yan et al. 2011; Brunner et al. 2016). The lack of knowledge about resistance mutations occurring outside of the CYP51 gene limit our understanding of the likely importance of interactions among resistance mutations occurring in other genes. Genome-wide analyses of fungicide resistance will fill important gaps in our understanding of how resistance is acquired within species.
Knowledge of where resistance genes are located in the genome is needed to integrate information on standing genetic variation and evidence for recent selection. Genomewide analyses led to the discovery of specific structural variation and single nucleotide polymorphisms (SNPs) underpinning fungicide resistance. A series of studies in Candida albicans established the contributions of variation in gene copy number (Selmecki et al. 2010), mutations in transcription factors (Coste et al. 2006; Dunkel et al. 2008), aneuploidy (Hill et al. 2013), and specific polymorphisms in over 240 genes (Ford et al. 2015) to fungicide resistance. Population genomic analysis of 24 environmental and clinical strains of Aspergillus fumigatus revealed segregating azole-resistance alleles in different genetic backgrounds (Abdolrasouli et al. 2015). In the agricultural environment, the emergence of fungicide resistance is expected to be rapid (McDonald and Linde 2002; Croll and McDonald 2017) as a result of the genetic homogeneity of host plants and intensive fungicide usage (Stukenbrock and McDonald 2008). In addition to rare de novo mutations, the standing genetic variation from natural pathogen populations is a likely source for fungicide adaptation that is seldom explored (Barrett and Schluter 2008; Yamashita and Fraaije 2018). Very few studies have considered the genomic landscape of natural populations when investigating the evolution of fungicide resistance in agroecosystems (Mohd-Assaad et al. 2016; McDonald et al. 2019).

The rise of resistance in agroecosystems is usually preceded by intense fungicide applications. For example, DMI fungicides were introduced in 1977 for use on cereals in Europe (Wyand and Brown 2005). After 20 years of low intensity applications, resistance was not detected in 1997 in a Swiss population of the wheat pathogen Mycosphaerella graminicola now called Zymoseptoria tritici (Gisi et al. 1997). However, two populations of the same wheat pathogen sampled in Oregon, United States, showed clear shifts in resistance against DMls after intensive fungicide use with resistance alleles being undetectable in 1992 but at high frequency in 2012 (Estep et al. 2015). Most European varieties of wheat are susceptible to the necrotrophic pathogen Parastagonospora nodorum (Downie et al. 2018) leading to the application of high amounts of fungicides to control this and other foliar diseases (Fones and Gurr 2015). The fungicide application impacts an entire community of wheat pathogens (Blixt et al. 2010; Knorr et al. 2019).

The haploid fungus $P$. nodorum negatively impacts wheat production worldwide (Oliver et al. 2012; Ficke et al. 2018). P. nodorum colonizes leaves and ears of wheat, causing necrotic lesions, and reducing yield. P. nodorum spreads across regions on contaminated seeds and wheat straw (Solomon et al. 2006; Bennett et al. 2007). The main migration routes among China, Europe, North America, and Australia were described in earlier studies (Stukenbrock et al. 2006). Most populations are characterized by frequent sexual recombination (Keller 
et al. 1997; Sommerhalder et al. 2006). A recent population survey in North America identified two major populations of $P$. nodorum with different genomic regions enriched in effectors under selection (Richards et al. 2019). This study and a second study (Pereira et al. 2020) show that $P$. nodorum readily responds to selection in the agricultural environment. European populations of $P$. nodorum sampled between 1994 and 2005 harbor point mutations in major genes related to fungicide resistance including in CYP51 (Blixt et al. 2009; Pereira et al. 2017). However, the genome-wide genetic architecture associated with azole resistance emergence remains largely unexplored.

In this study, we analyze a collection of 159 P. nodorum genomes from seven field populations collected around the world and perform genome-wide association studies (GWAS) to establish the genetic basis of early fungicide sensitivity globally. All analyzed genomes date from before intensive fungicide application was common practice at most sampling sites. We also investigate whether the emergence of fungicide resistance led to pleiotropic effects using measures of fungal growth and melanization.

\section{Materials and Methods}

\section{Fungal Populations}

Isolates of $P$. nodorum were sampled from wheat fields naturally infected by the pathogen. A total of 159 isolates chosen from seven fields ( $\sim 20$ isolates per field) were included in our analyses. The sampled countries/regions included Australia (2001), Iran (2005 and 2010), South Africa (1995), Switzerland (1999A and 1999B), New York (United States, 1991), Oregon (United States, 1993), and Texas (United States, 1992). All isolates were previously genotyped using microsatellite markers (Stukenbrock et al. 2006; McDonald et al. 2012). We selected only unique haplotypes for this study.

In earlier publications (Sommerhalder et al. 2006; Stukenbrock et al. 2006; McDonald et al. 2012, 2013; Pereira et al. 2017, 2020), the Switzerland 1999B population was indicated to originate from China in 2001. As a result of the genome sequence analyses reported in this paper, we believe that a transcription error led to mislabeling of the China 2001 population, which we now believe originated from a Swiss field of wheat located near Bern, $\sim 150 \mathrm{~km}$ away from where the Swiss 1999A population was collected. The re-assignment of the China 2001 population to Switzerland 1999B does not compromise any of the analyses or interpretations reported in this manuscript.

\section{Fungicide Sensitivity Phenotyping}

Isolates were recovered from long-term storage in silica gel at $-80^{\circ} \mathrm{C}$ by placing silica gel fragments on the center of round Petri dishes containing potato dextrose agar (PDA, $4 \mathrm{~g} \mathrm{I}^{-1}$ potato starch, $20 \mathrm{~g} \mathrm{I}^{-1}$ dextrose, $15 \mathrm{~g} \mathrm{I}^{-1}$ agar, and $50 \mathrm{mg}$ $\mathrm{I}^{-1}$ kanamycin). The plates were placed in chambers with a constant temperature of $24^{\circ} \mathrm{C}$ in the dark to induce mycelial development. After 3 days of growth, mycelium from each isolate was excised from the edges of the colonies with a cork borer $(5 \mathrm{~mm})$ and transferred to new PDA plates, to be used as the inoculum source for the sensitivity phenotyping experiment. All 159 isolates were phenotyped using four doses of propiconazole (Syngenta, Basel, Switzerland) chosen based on previous experiments that were conducted to determine the dose range that revealed the greatest variation in sensitivity among isolates. The selected doses were $0,0.1,0.5$, and $1 \mathrm{ppm}$ of propiconazole diluted in dimethyl sulfoxide (DMSO, $0.002 \% \mathrm{v} / \mathrm{v}$ ). The doses of propiconazole or DMSO alone (as a control) were incorporated into molten PDA $\left(\sim 50^{\circ} \mathrm{C}\right)$ with a magnetic stirrer and a $50 \mathrm{ml}$ volume was poured into square Petri dishes $(120 \mathrm{~mm} \times 120 \mathrm{~mm} \times 17 \mathrm{~mm}$, HUBERLAB).

Using a $5 \mathrm{~mm}$ cork borer, mycelial plugs were excised from the edge of colonies developing after 7 days of growth on the inoculum plates. Four plugs from each isolate were placed in the corners of square plates with equidistant separation. Isolates were replicated twice, generating eight colonies in total for each of the four doses. Plates were randomized in an incubation chamber and grown at a constant temperature of $24^{\circ} \mathrm{C}$ and with no light during the entire experiment. Digital images of the colonies were acquired at 8 days after inoculation through each plate lid. Images were analyzed using a batch script in ImageJ (Schneider et al. 2012; Lendenmann et al. 2014) matching parameters used in Pereira et al. (2020). The method yielded quantitative measures of each colony for total colony area $\left(\mathrm{mm}^{2}\right)$ and melanization (mean gray values). The effective concentration that inhibited mycelial growth by $50 \%$ ( $\mathrm{EC}_{50}$ ) was determined using a dose-response curve based on colony radius ( $\sqrt{\text { total area } / \pi})$ values in the R package $d r c$ version 3.0-1 (Ritz et al. 2015).

\section{Whole-Genome Sequencing}

Fragments of mycelium from 4-day-old colonies growing on PDA media were transferred to $50 \mathrm{ml}$ Potato Dextrose Broth (PDB) media and cultured for $4-6$ days at $24^{\circ} \mathrm{C}$ while shaking at $120 \mathrm{rpm}$. The resulting mycelial colonies were filtered through sterile cheesecloth and lyophilized for $72 \mathrm{~h}$. Dried fungal material was used for DNA extraction with the DNeasy Plant Mini Kit (Qiagen) following the manufacturer's standard protocol. We sequenced the genomes for all 159 isolates included in this study. The sequencing was performed on an Illumina HiSeq 2500 platform producing paired-end reads of $150 \mathrm{bp}$. Preparation of sequencing libraries and sequencing was performed at the Functional Genomics Center in Zurich. Raw sequence reads were deposited in the $\mathrm{NCBI}$ Short Read Archive under BioProject PRJNA606320. 
Genome Alignment, Variant Calling, and Quality Filtering

Raw reads were trimmed for remaining Illumina adaptors and read quality was assessed using Trimmomatic version 0.36 (Bolger et al. 2014) with the following parameters: illuminaclip $=$ TruSeq3-PE.fa:2:30:10, leading $=10$, trailing $=10$, slidingwindow $=5: 10$, minlen $=50$. Trimmed reads were aligned against the reference genome established for the isolate SN2000 (Richards et al. 2017). Reference genome mapping was performed using the short-read aligner Bowtie2 version 2.3.3 (Langmead and Salzberg 2012), using the very-sensitive-local option. Picard tools version 2.17.2 was used to mark PCR duplicates (http://broadinstitute.github.io/ picard, last accessed July 2019). All sequence alignment (SAM) files were sorted and converted to binary (BAM) files using SAMtools version 1.2 (Li et al. 2009). SNP calling and variant filtration were performed using the Genome Analysis Toolkit (GATK) version 3.8-0 (McKenna et al. 2010). Initially, we used HaplotypeCaller on each isolate BAM file individually with the -emit-ref-confidence GVCF and -ploidy 1 options. Then, joint variant calls were produced using GenotypeGVCFs with the flag -maxAltAlleles 2. Finally, SelectVariants and VariantFiltration was used for hard filtering SNPs with the following cut-offs: QUAL < 200; QD < 10.0; MQ < 20.0; $2>$ BaseQRankSum $>2$; - 2>MQRankSum > 2; $2>$ ReadPosRankSum $>2$. SNPs that failed the PASS designation by GATK were removed and we kept only bi-allelic sites. For the final data set, we retained SNPs with a genotyping rate of at least $90 \%$ and a minimum allele frequency of $5 \%$ using vcftools version 0.1.15 (Danecek et al. 2011).

\section{Genome-Wide Association Mapping}

Association analysis was performed using the $\mathrm{R}$ package GAPIT version 2 (Tang et al. 2016), using a mixed linear model (MLM) (Yu et al. 2006). This model improves the control of false positives (type I errors) by incorporating fixed and random effects. Alternatively, we tested the inclusion of principal components (PCS) from a PC analysis to correct for population structure $(\mathrm{Q})$ or a kinship matrix $(\mathrm{K})$ to account for cryptic relationships (Yu et al. 2006; VanRaden 2008). We identified the most appropriate set of parameters and covariates by comparing the models MLM $+\mathrm{K}$ and MLM $+\mathrm{K}+\mathrm{Q}$, where $\mathrm{Q}$ stands for the three first PCs. Based on a Bayesian information criterion (Schwarz 1978) analysis performed in GAPIT, the MLM + K model was selected as the most appropriate for our data set. We considered associations to be significant when $P$ values were smaller than the Bonferroni threshold at $\alpha=0.05(P<1.1 \mathrm{e}-07)$. False discovery rate (FDR) thresholds of $5 \%(P<7.15 \mathrm{e}-07)$ and $10 \%$ $(P<8.26$ e-06) were determined using the $\mathrm{R}$ package $q$ value version 2.18.0 (Storey and Tibshirani 2003). We explored the genomic regions containing significantly associated loci using bedtools version 2.29.0 (Quinlan and Hall 2010).
Population Structure and Linkage Disequilibrium Analyses Population structure was inferred using both a PC analysis in TASSEL version 5.2.56 and a model-based clustering implemented in STRUCTURE v.2.3.4 (Pritchard et al. 2000; Bradbury et al. 2007). We visualized the two first PCs using the ggplot2 package in $\mathrm{R}$. The genetic markers used as input in STRUCTURE were composed of 2,348 SNPs. These SNPs were selected randomly across the genome using a sampling window of $10 \mathrm{~kb}$ to ensure no/very low linkage disequilibrium (LD) among loci. We chose an admixture model independent of prior population information and with correlated allele frequencies. The algorithm ran with a burn-in length of 50,000 and a simulation length of 100,000 Markov chain Monte Carlo repetitions. We varied estimations of $K$ between 1 and 10 , with 10 repetitions per $K$. The most likely number of populations ( $K$ ) was estimated based on Evanno's method (Evanno et al. 2005) implemented using the $\mathrm{R}$ package pophelper version 2.3.0 (Francis 2017). Regions in the genome spanning the most significant associations were further investigated in detail for signatures of LD. Using the vcftools option -hap-r2, we compared all possible SNP pairs in a $5 \mathrm{~kb}$ window. A heatmap was produced based on the $r^{2}$ values using the R package LDheatmap version 0.99-7 (Shin et al. 2006).

\section{Allelic Effect and Trade-Off Analyses}

We used GAPIT (Tang et al. 2016) for estimations of allelic effects. Allelic effects on $\mathrm{EC}_{50}$ values were compared with allelic effects on growth rate and melanization (under temperatures of 18,24 , and $30^{\circ} \mathrm{C}$ ). The total fungicide resistance variation explained by each SNP was determined using a linear mixed-effect model implemented in the Ime4 R package version 1.1-19 (Bates et al. 2015). EC 50 values were used as response variables, the SNPs as fixed effects and populations were included as random effects. Using the function r.squaredGLMM from the MuMln package version 1.43 .6 in $R$ we obtained $R^{2}$ indices (Nakagawa and Schielzeth 2013).

\section{Homology Analyses of Candidate Genes}

Amino acid sequences of all genes in the $P$. nodorum SN15 reference genome were obtained from the UniProt database under the proteome ID 000001055 (Hane et al. 2007; Magrane and Consortium 2011). Gene annotations for the SN2000 reference genome are not available in databases, hence we aligned the predicted protein sequences from the SN15 assembly retaining queries with a 95\% minimum identity score using the software exonerate version 2.2.0 (Slater and Birney 2005; Richards et al. 2017). For inferences on gene function, we identified conserved domains using InterProScan v.5.44-79, NCBI Conserved Domain v.3.17, and HMMER v3.3 database search tools (Quevillon et al. 2005; Finn et al. 2011; Marchler-Bauer et al. 2015). 

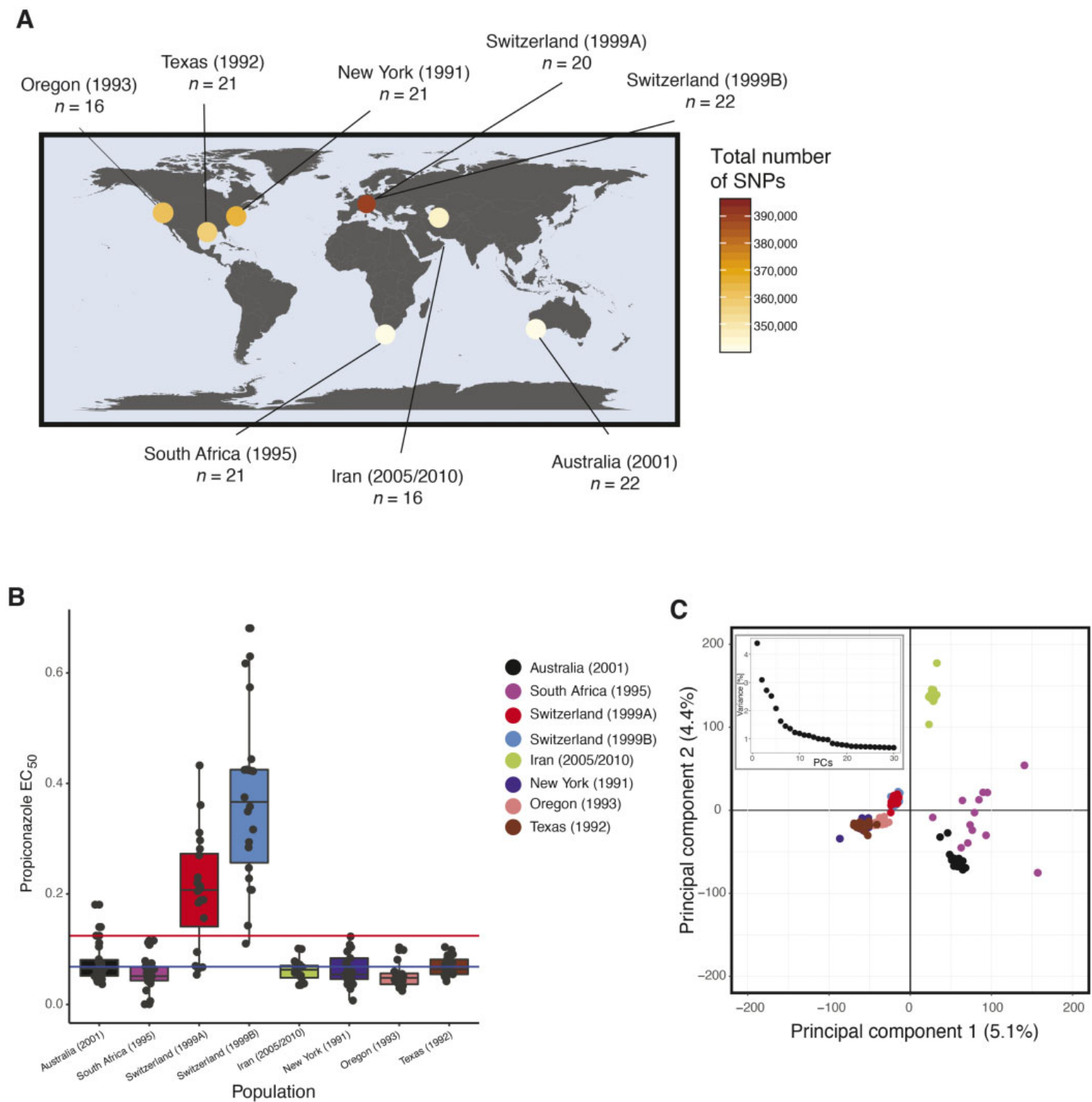

FIG. 1.-Geographic origins of the 159 P. nodorum isolates. (A) World map showing sampling sites, number of isolates per population and the number of SNPs. $(B)$ Boxplots of $\mathrm{EC}_{50}$ (in ppm) values for each isolate in each population. The red line shows the mean overall $\mathrm{EC}_{50}$ and the blue line indicates the overall median EC 50. (C) The first two PCs from a PCA of 436,365 genome-wide SNP genotypes, and a box with the explained variance across the 30 first PCs. Populations are color-coded.

\section{Results}

\section{Population Level Differences for Fungicide Sensitivity and Genetic Diversity}

We analyzed sensitivity to an azole fungicide in a worldwide collection of 159 P. nodorum isolates using individual $\mathrm{EC}_{50}$ measures. The pathogen strains came from seven field populations located in Australia $(n=22)$, South Africa $(n=21)$, Switzerland 1999A ( $n=20)$, Switzerland 1999B $(n=22)$, Iran $(n=16)$, New York $(n=21)$, Oregon $(n=16)$, and Texas $(n=21) \quad$ (fig. $1 \mathrm{~A}$ and supplementary table S1, Supplementary Material online). In total, 39 isolates (24.5\%) had $\mathrm{EC}_{50}$ values higher than the overall average of $0.12 \mathrm{ppm}$. The populations from Switzerland showed the highest average $\mathrm{EC}_{50}$ values $(0.20$ and $0.37 \mathrm{ppm}$ in $1999 \mathrm{~A}$ and $1999 \mathrm{~B}$, respectively; $P \leq 0.01$; fig. $1 B$ and supplementary fig. $S 1$, Supplementary Material online) whereas the population from Oregon had the lowest average $\mathrm{EC}_{50}(0.05 \mathrm{ppm})$. We sequenced genomes for all 159 isolates using Illumina shortread sequencing. On average, we obtained a mean sequencing depth of $24 \times$ per individual, with a SNP density of approximately 12 SNPs per kb. After removing SNPs with more than $10 \%$ missing genotypes and minor allele frequencies $<5 \%$, we retained a total of 436,365 SNPs to be used for 

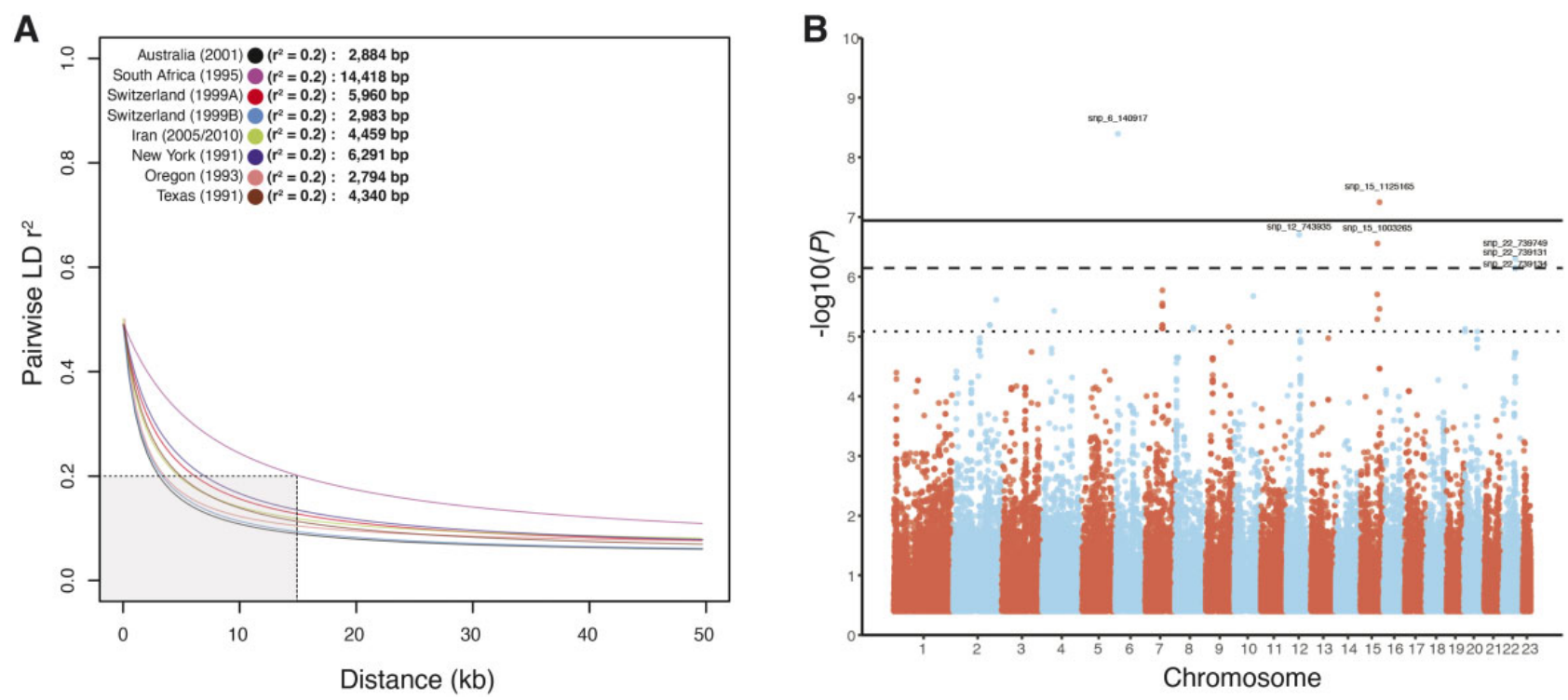

FIG. 2.-LD decay in each population and Manhattan plot of GWAS for fungicide sensitivity. (A) Pairwise LD decay among all SNPs within a fixed window of $50 \mathrm{~kb}$ for each population. A nonlinear model was fitted based on $r^{2}$ measures along with the first $50 \mathrm{~kb}$ on chromosome 1 using the equation of Ingvarsson (2005). The gray shading indicates the total area needed for all populations to reach $r^{2}=0.2$. (B) Manhattan plot showing the SNP associations with fungicide resistance. SNP markers are shown as dots colored according to their associated chromosomes. Different significance levels are displayed on the $y$-axis: Horizontal lines represent the thresholds for FDR 10\% (dotted line), FDR 5\% (dashed line), and after Bonferroni correction (solid line). SNPs above FDR $5 \%$ were labeled with a specific identifier (chromosome number + SNP coordinates in bp).

downstream analyses. The total number of SNPs retained per population varied from 340,929 in Australia to 395,054 in Switzerland 1999B. LD decayed differently among populations (fig. 2A). In the populations from Australia, Switzerland, Iran, and the United States (New York, Oregon, Texas), $r^{2}$ reached values below 0.2 within $10 \mathrm{~kb}$. In the population from South Africa $r^{2} \sim 0.2$ was reached at $15 \mathrm{~kb}$.

A population structure analysis revealed clusters of isolates differentiated according to the continent of origin (fig. 1C and supplementary fig. S2, Supplementary Material online). A major cluster was formed by isolates from Switzerland and the United States (New York, Oregon, Texas). Isolates from Australia, South Africa, and Iran constituted a second and more dispersed cluster. Incorporating a third PC, isolates remain grouped according to their continent of origin, except for the South African becoming more dispersed (supplementary fig. S2, Supplementary Material online). We analyzed the clustering scenarios of $K=2$ and $K=3$ (supplementary fig. S3 and table S2, Supplementary Material online). At $K=2$, Australia and South Africa belonged to cluster 1, whereas the other populations composed cluster 2. At $K=3$, the Iranian population constituted most of cluster 3 which was shared with genotypes from Switzerland and South Africa (supplementary fig. S4, Supplementary Material online). The global dispersal of $P$. nodorum was proposed to mirror the domestication and expansion of the wheat host (Heun 1997; Balter 2007; McDonald et al. 2012; Balfourier et al. 2019).
Wheat originated in the Fertile Crescent and then spread across Europe and Asia for thousands of years before Europeans brought it to the American continent $\sim 500$ years ago and Australia $\sim 200$ years ago. P. nodorum is a seedborne pathogen, so it is likely that the pathogen moved globally on infected wheat seed. Because the Iranian P. nodorum population is closest to the Fertile Crescent, we expect it would have retained most of the ancestral polymorphism. This is reflected by the finding that it was a hotspot of genetic diversity detected previously by microsatellite markers (McDonald et al. 2012) and neutral SNP markers (Pereira et al. 2020). In Australia, strict quarantine measures likely limited the introduction of the pathogen on infected wheat material (Oliver et al. 2012). Consistent with this proposed bottleneck, we found that the Australian population had low diversity and was distinct from other populations.

\section{Genetic Architecture of Fungicide Sensitivity across Populations}

To unravel the genetic architecture of fungicide resistance in $P$. nodorum, we performed genome-wide association analyses using all 159 isolates. We associated genotypes at the 436,365 SNP markers with the $\mathrm{EC}_{50}$ phenotypes and identified 34 SNPs significantly associated with fungicide resistance (supplementary table S3, Supplementary Material online). Two associations above the most stringent threshold (Bonferroni $\alpha=0.05, P<1.1 \mathrm{e}-07$ ) were located on chromosomes 6 and 15 (fig. 2B). At the FDR 5\%, we found five 
additional associations on chromosomes 12, 15, and 22. At FDR $10 \%$, we identified a total of 27 additional SNPs on chromosomes 2, 4, 7, 8, 9, 10, 15, and 20 (fig. 2B). The average distance between genes in the $P$. nodorum genome is $1.2 \mathrm{~kb}$ (Syme et al. 2018). We considered SNPs to be in close proximity if they were located within $1 \mathrm{~kb}$ of the closest gene. We found 5 associations $>1 \mathrm{~kb}$ from the nearest gene and 16 within $1 \mathrm{~kb}$ of a gene. Thirteen associations were located within a gene (supplementary table S3, Supplementary Material online). Quantile-quantile (QQ) plots showed there was not meaningful inflation due to population structure using the $\mathrm{MLM}+\mathrm{K}$ model (supplementary fig. S5, Supplementary Material online).

Populations differed in their complement of fungicide resistance mutations. Two SNPs above the Bonferroni threshold (snp_6_140917 and snp_15_1125165) were both present in the populations from Switzerland and Texas but were absent in South Africa. The mutation underlying the third strongest association (snp_12_743935, FDR 5\%) was exclusively present in the populations from Switzerland but was missing in all other populations. Taken together, based on the top seven SNPs passing the $5 \% \mathrm{FDR}$, all populations carried at least one resistance mutation with the exception of South Africa.

\section{Genomic Context of the Key Loci Contributing to Azole Sensitivity}

We investigated the genomic context of the most strongly associated SNPs. The strongest association was snp_6_140917 on chromosome $6\left(P=4.03 * 10^{-9}\right.$, supplementary fig. S6, Supplementary Material online). This SNP was located 1,044 bp upstream of the nearest gene (SNOG_15057), which encodes a helix-loop-helix (HLH) domain functioning as a transcription factor (supplementary table S3, Supplementary Material online). HLH-domain proteins constitute a large family of proteins acting as gene expression regulators (Massari and Murre 2000). Some members of this family were shown to boost drug resistance gene expression in human tumors (Vandeputte et al. 2002; Cheung 2004) and plant pathogens (Liu et al. 2015). The second most strongly associated genomic region was on chromosome 15 (fig. 3A). The SNPS snp_15_1125165 $\left(P=5.65 * 10^{-8}\right)$, and snp_15_1124326, $\left(P=3.45 * 10^{-6}\right)$, were located in close proximity at chromosomal positions 1.125 and $1.124 \mathrm{Mbp}$, respectively (fig. 3A). The SNP at $1.125 \mathrm{Mbp}$ comprised a nonsynonymous mutation (threonine to isoleucine) in the gene SNOG_14185 (supplementary fig. S7, Supplementary Material online). The SNP at $1.124 \mathrm{Mb}$ comprised an intron mutation in the same gene. SNOG_14185 encodes a transmembrane transporter and belongs to the major facilitator superfamily (MFS) with similarity to the Yeast Polyamine transporter 1 (Tpo1). MFS transporters are known multidrug resistance components in model organisms and fungal pathogens (De Rossi et al. 2002). The chromosomal regions surrounding snp_6_140917 and snp_15_1125165 show low LD $\left(r^{2}<0.2\right)$ with each region harboring only a single gene (supplementary fig. S6A, Supplementary Material online and fig. 3A, respectively).

\section{Combinatorial Effects of Fungicide Resistance Loci}

We evaluated how the frequencies and effects of the individual SNP associations contributed to the overall azole resistance of $P$. nodorum. The resistance allele at snp_6_140917 was present in $41 \%$ of isolates from Switzerland 1999B, in 10\% of the New York isolates and in $10 \%$ of the Texas isolates (supplementary fig. S8A, Supplementary Material online). The global frequency was about $8 \%$ across all 159 isolates. The resistance allele at snp_15_1125165 was present at a global frequency of $14 \%$, at $5 \%$ in Australia, $15 \%$ in Switzerland 1999A, 55\% in Switzerland 1999B, 19\% in Iran, 6\% in Oregon, and $5 \%$ in Texas (supplementary fig. S8B, Supplementary Material online). When comparing the degree of fungicide sensitivity, the group of isolates containing either of the two resistance alleles had higher $\mathrm{EC}_{50}$ values (fig. $3 B$ and supplementary fig. S6, Supplementary Material online). At the population level, the group of isolates harboring the resistant allele at snp_6_140917 was significantly more resistant only within Switzerland 1999B (t-test $P=0.004$; supplementary fig. S6, Supplementary Material online). For snp_15_1125165, the group of isolates containing the resistant allele was more resistant within Switzerland 1999A ( $t$-test $P=0.03$ ) but only marginally more resistant in Switzerland 1999B ( $t$-test $P=0.08$, fig. 3C). In contrast, in the populations from Australia, Iran, and Oregon, there were no significant differences between isolates carrying the different alleles.

We expanded the comparisons with genotypes differentiated by nonsynonymous mutations in the CYP51 gene. Isolates with a nonsynonymous CYP51 resistance mutation in the background and a resistance allele identified by GWAS showed a significant increase in resistance in 4 out of 5 combinations (fig. 4). However, when considering only the resistance alleles detected by the GWAS, while disregarding the CYP51 resistance mutations in the genetic background, we did not find a significant increase in resistance. We performed a second GWAS after excluding isolates carrying the CYP51 nonsynonymous resistance mutations and we found no significant associations at genome-wide significance thresholds. Next, we assessed the individual contributions of the identified resistance alleles to the overall variation in fungicide sensitivity among populations. The mutations identified in the CYP51 gene contributed $63.2 \%$ of the total phenotypic variation whereas the snp_15_1125165 in the MFS transporter gene contributed only $6.1 \%$ of the phenotype variation. Despite the major effect of CYP51 haplotypes on resistance, we found that individual CYP51 SNPs were associated only at a FDR $10 \%$ for the strongest associations (supplementary table S3, Supplementary Material online). 
A

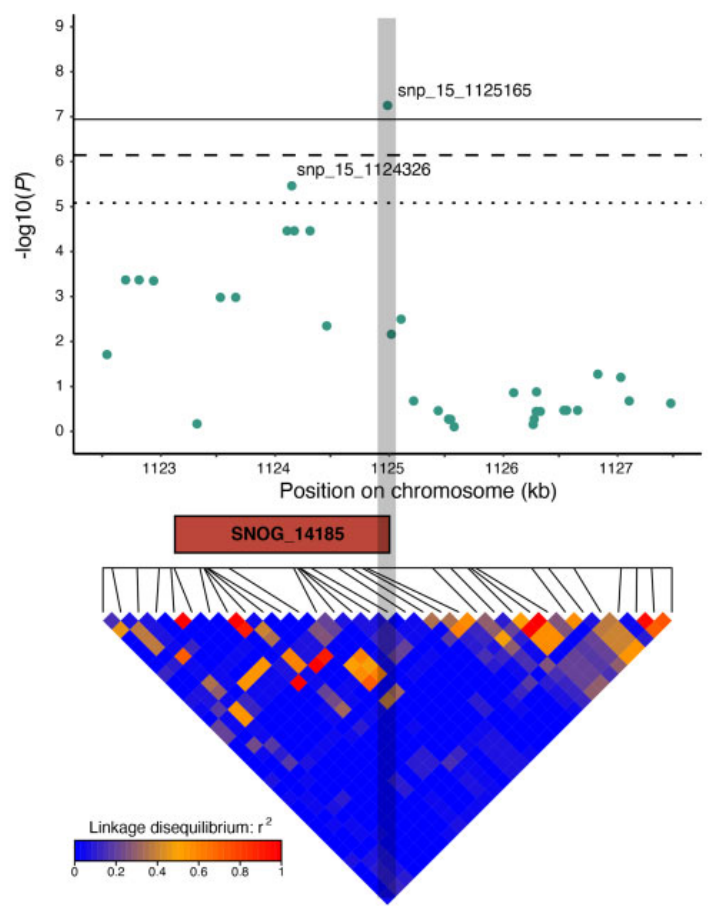

B

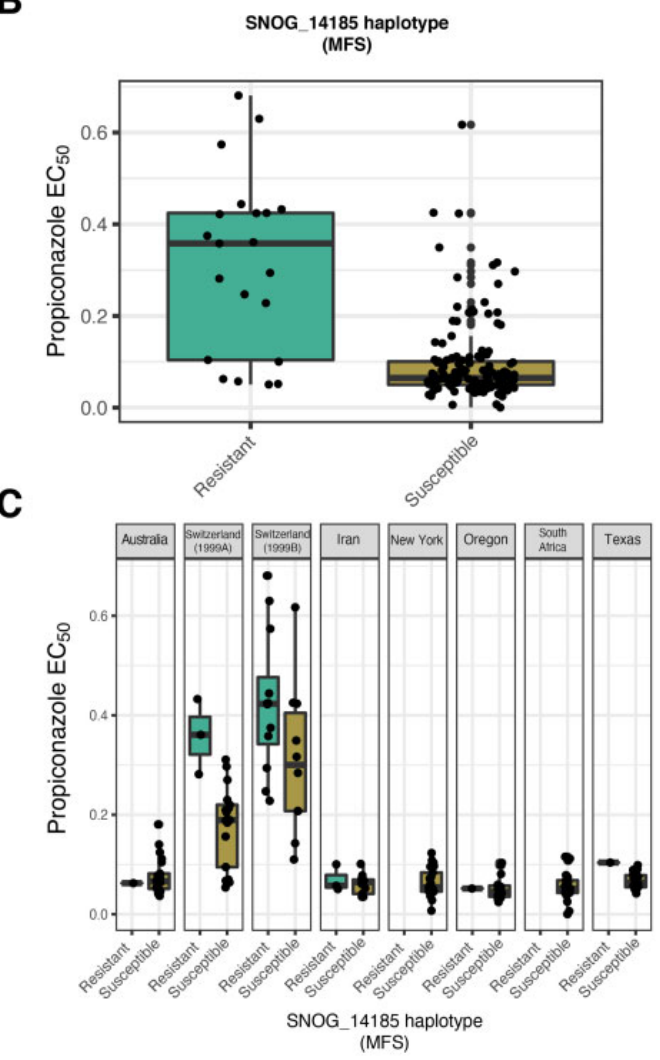

FIG. 3.-Analysis of the SNP associations near the MFS transporter gene SNOG_14185 associated with azole resistance. (A) Top panel: Scatter plot for association $P$ values of SNPs within a $5 \mathrm{~kb}$ region centered on the peak snp_15_1125165. Horizontal lines represent the thresholds for FDR $10 \%$ (dotted line), FDR 5\% (dashed line), and after Bonferroni correction (solid line). SNOG_14185 encoding an MFS transporter is shown in orange. Bottom panel: LD map for the pairwise comparison among SNPs within a $5 \mathrm{~kb}$ window. (B) Boxplots showing $\mathrm{EC}_{50}$ values (in ppm) for propiconazole among global isolates carrying the resistant or susceptible allele at snp_15_1125165. (C) Boxplots showing EC 50 values in isolates carrying the resistant or susceptible allele at snp_15_1125165 organized according to population.

This is possibly explained by high LD in the chromosomal region and relatively minor contributions of individual mutations to resistance (supplementary fig. S9, Supplementary Material online).

\section{Allelic Effects Associated with Fungicide Resistance and Testing for Trade-Offs}

Allelic effects quantify the mean difference in phenotypic values between genotypes carrying either of two alleles at a locus. We investigated the correlation of allelic effects between fungicide sensitivity and six quantitative life history traits including growth and melanization at the temperatures 18,24 , and $30^{\circ} \mathrm{C}$ (fig. 5). Melanin is an important secondary metabolite in microbes and is broadly related to host colonization and survival under stressful conditions such as UV radiation, heat stress, and antimicrobial compounds (Rosas et al. 2000; Nosanchuk and Casadevall 2006; Lendenmann et al. 2015). Melanin was previously shown to be associated with fungicide resistance in the plant pathogen $Z$. tritici (Lendenmann et al. 2014). We focused on the seven most significant SNPs identified in the GWAS for fungicide resistance and performed association mapping analyses for the six other traits. Using allelic effect correlations, we investigated whether resistance mutations showed evidence for pleiotropic effects on any other trait. We found that the most significant SNPs had no meaningful impact on any other analyzed traits (fig. $5 A$ and $B$ ) and we found no strong correlation between allelic effects of fungicide resistance and the other traits (fig. 5C).

\section{Discussion}

We used whole-genome sequencing data and association mapping to reveal the multilocus genetic architecture of fungicide resistance in $P$. nodorum. We identified significant differences in azole resistance among a global set of field populations. Some populations evolved distinct combinations of resistance alleles which showed interactions when cooccurring in a same genetic background. We identified several significantly associated SNPs in close proximity to candidate resistance genes, including an MFS transporter. There 


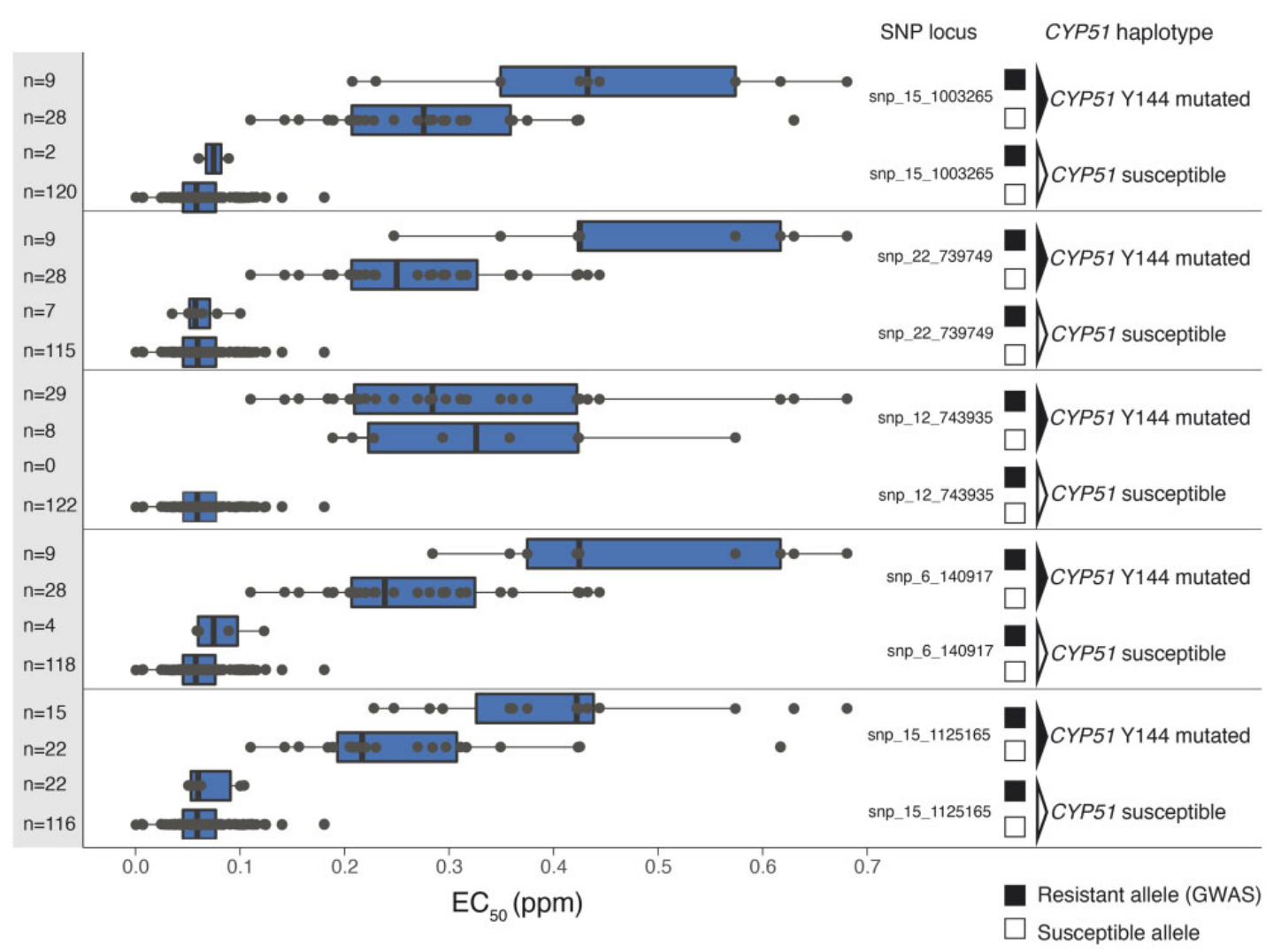

FIG. 4.-Comparisons of $\mathrm{EC}_{50}$ values among resistance haplotypes. Combinatorial genotypes of mutations in the CYP51 gene and the most significant associations found by GWAS are shown. The number of isolates carrying a specific genotype is indicated on the $y$-axis. On the right side, the CYP51 haplotype column identifies genotypes by their nonsynonymous Y144 mutations. Contrasting resistant and susceptible CYP51 haplotypes. The SNP locus column identifies the resistant (black square) versus the susceptible (empty square) at SNP loci identified by GWAS.

was no evidence for trade-offs associated with the observed resistance to azoles.

The genetic basis of fungicide resistance includes both qualitative and quantitative factors (de Waard et al. 2006). The presence or absence of a sensitive target site is typically considered a qualitative factor [e.g., Strobilurus tenacellus and strobilurin A \{Kraiczy et al. 1996\}]. Previous studies oriented around single known loci identified major genetic determinants (e.g., qualitative factors) associated with fungicide resistance in P. nodorum (Blixt et al. 2009; Pereira et al. 2017). Quantitative factors are often associated with a number of different mechanisms that make minor contributions to overall resistance. In this study, we used a genome-wide approach to identify both major and minor contributions to resistance. We found 34 candidate loci distributed across the genome, including the CYP51 gene, underlying quantitative variation in fungicide sensitivity across populations. In clinical resistance studies, an increasing number of genetic loci affecting drug resistance have been described in viruses, protozoa, and bacteria (Manolio 2013; Chewapreecha et al. 2014; Holt et al. 2015; Power et al. 2017). The emergence of fungicide resistance in plant pathogenic fungi has been associated mainly with mutations in genes encoding the targeted protein.
However, GWAS based on whole-genome sequencing in the barley scald pathogen Rhynchosporium commune (Mohd-Assaad et al. 2016) showed that $R$. commune evolved resistance to azoles via a combination of genetic variants in addition to mutations in the CYP51 gene.

The evolution of azole resistance in $P$. nodorum was likely initiated by mutations in the CYP51 gene coupled with more recent mutations in other loci. In both $P$. nodorum and $R$. commune, the mutations with the greatest impact on azole resistance were found in the CYP51 gene (Mohd-Assaad et al. 2016). The convergent evolution of azole resistance based on CYP51 mutations is a major feature of azole resistance globally (Fisher et al. 2018). However, additional loci may elevate fungicide resistance in a subset of populations. There is growing evidence that herbicide resistance in plants involves sites that are not targeted by the herbicide (Baucom 2019). These nontarget sites are usually related to herbicide translocation or detoxification (Peng et al. 2010; Leslie and Baucom 2014).

We observed different levels of resistance and combinations of resistance alleles among our worldwide populations. Such a mosaic structure in resistance factors was also observed among populations of Streptococcus pneumoniae and Mycobacterium tuberculosis (Chewapreecha et al. 
A

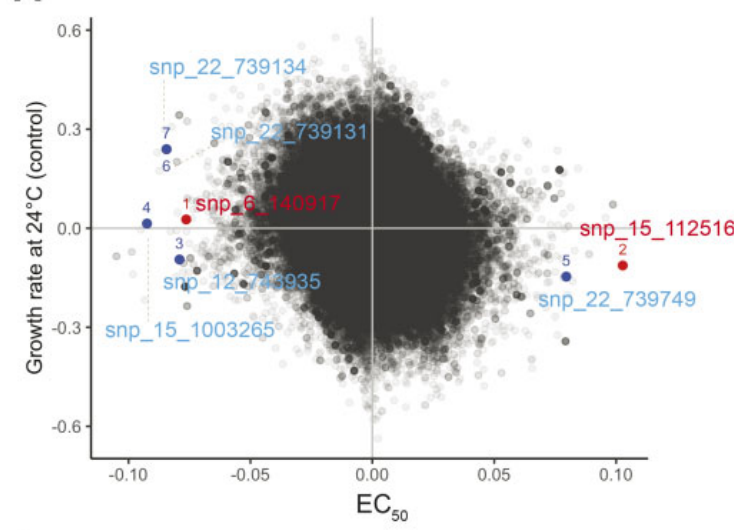

B

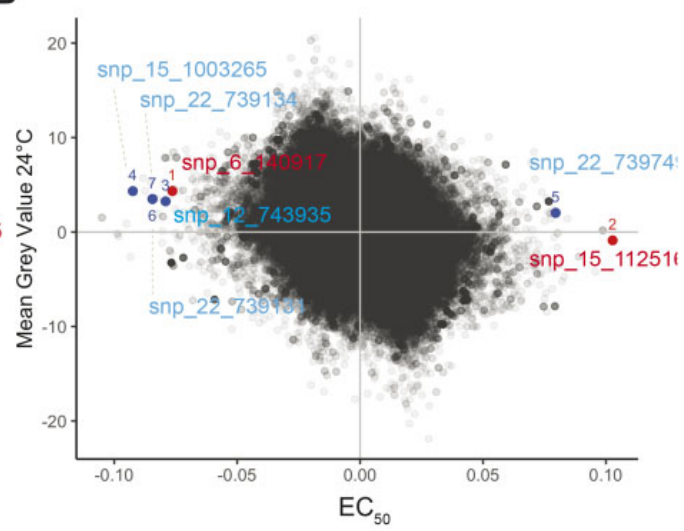

\section{C}

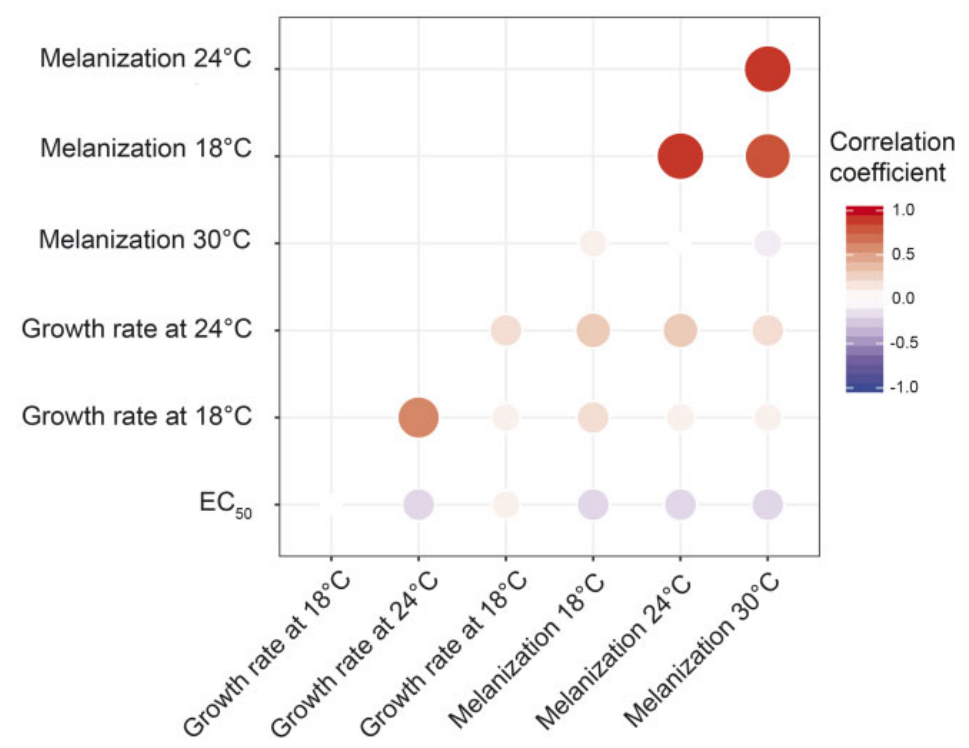

FIG. 5.-Genome-wide allelic effect correlations. Allelic effects for $\mathrm{EC}_{50}$ and $(A)$ growth rate and $(B)$ melanization at $24^{\circ} \mathrm{C}$. SNPs that were significantly associated with propiconazole sensitivity are indicated. (C) Mean allelic effect correlation coefficients for $\mathrm{EC}_{50}$, growth rate $\left(\mathrm{mm}\right.$ day $\left.{ }^{-1}\right)$, and melanization at different temperatures. Sizes of circles represent degrees of significance and the opacity of colors are proportional to the size of the correlation coefficient.

2014; Farhat et al. 2019), with certain populations enriched in particular resistance determinants (Chewapreecha et al. 2014). The highest number of resistance alleles was found in $P$. nodorum isolates from Switzerland (both 1999A and 1999B), which were also the most resistant populations. The use of azoles in Europe started in 1979 (Estep et al. 2015). We postulate that the Swiss populations were either directly selected for fungicide resistance or received resistance alleles through gene flow from areas where fungicides were intensively used. Considering migration patterns based on microsatellite markers (Stukenbrock et al. 2006), it is likely that resistance genotypes will be exchanged among populations. Populations of the barley pathogen $R$. commune showed a similar pattern, with the Swiss population among the most resistant worldwide and an exchange of migrants among continents (McDonald 2015; Mohd-Assaad et al. 2016). Broad usage of DMls in Australia only started in 2002 to control wheat diseases (Wellings 2007). In South Africa foliar application of DMI was introduced in 1988 for barley pathogens (Campbell and Crous 2002). Therefore, the lower selection pressure imposed by the fungicide application regime was likely insufficient for the widespread emergence of resistant genotypes or an increase in base-line resistance in populations. Given that we analyzed populations many years after the onset of widespread fungicide applications, many mutations affecting azole resistance may have arisen in these populations.

We identified a potentially new mechanism of azole resistance in $P$. nodorum. Isolates with the lowest sensitivity to propiconazole often harbored resistance mutations at both the CYP51 locus and the MFS transporter locus. MFS transporters are among the largest protein families (Stergiopoulos et al. 2002), ubiquitous in the cell membrane of prokaryotes (Hirai and Subramaniam 2004), and eukaryotes (Henderson 
and Maiden 1990). These transporters contribute to cell-tocell communication as well as movement of pathogenicity toxins and antimicrobial drugs through the cell membrane (Paulsen et al. 1996). Importantly, MFS transporters can also act as efflux pumps that reduce intracellular drug concentrations (Kretschmer et al. 2009; Omrane et al. 2015; Redhu et al. 2016). In the plant pathogens Botrytis cinerea and $Z$. tritici, upregulation of an MFS transporter was shown to reduce sensitivity to azole fungicides (Kretschmer et al. 2009; Omrane et al. 2017). In P. nodorum, we observed correlations between MFS mutations and azole sensitivity. MFS transporters can also vary in their substrate affinity as found for a multidrug transporter in C. albicans (Pasrija et al. 2007). Depending on the type of MFS transporter mutations, C. albicans varied in sensitivity to different drugs, including an azole. The nonsynonymous mutation we identified in $P$. nodorum could influence this MFS transporter's affinity for propiconazole. Interestingly, the group of isolates lacking CYP51 resistance mutations, but carrying the more resistant variant of the MFS transporter were highly susceptible, indicating that the MFS transporter mutations depend on CYP51 mutations to have an effect. This is similar to what was observed in Z. tritici (Omrane et al. 2015). Epistasis among resistance-encoding genes is also known from $C$. albicans (Hill et al. 2013; Ciudad et al. 2016) and appears to be a common phenomenon associated with the emergence of de novo resistance mutations.

A major constraint on the emergence of resistance mutations is negative pleiotropy. Fungicides generally target essential metabolic processes. By reducing the synthesis of ergosterol, azoles negatively impact cell fluidity and functions through membrane defects (Georgopapadakou and Walsh 1996; Lass-Flörl 2011). Resistance mutations are most successful if they confer decreased binding affinity with the fungicide but do not negatively impair normal protein functions (Karaoglanidis et al. 2001; Yan et al. 2011). Resistance mutations that lead to overproduction of targeted proteins may negatively impact the cellular energy budget (Lang et al. 2009). Hence, resistance mutations are likely to confer advantageous effects only in the presence of the fungicide. Fitness costs in the absence of the pesticide constrain the emergence of acquired resistance in plants, bacteria, and fungi (Schenk and de Visser 2013; Moura de Sousa et al. 2017; Pagnout et al. 2019). Interestingly, we found no evidence that the most important fungicide resistance mutations negatively impacted growth rates or melanization in $P$. nodorum. This is in contrast to other fungal pathogens such as $Z$. tritici and $R$. commune where growth rates were negatively affected in the absence of azoles (Lendenmann et al. 2015; MohdAssaad et al. 2016). Herbicide resistance shows a broader spectrum of pleiotropic effects among species (Powles and Yu 2010; Baucom 2019) with the most common mutations having limited fitness costs (Tranel and Wright 2002). Fitness costs of resistance mutations can also be reduced through compensatory mutations, as shown in bacteria (Levin et al. 2000; Trindade et al. 2009; Moura de Sousa et al. 2017) and postulated in fungi (Lucas et al. 2015; Dettman et al. 2017). We also found no evidence for genetic trade-offs between fungicide resistance and growth at different temperatures.

These findings suggest that $P$. nodorum either evolved azole resistance without relying on costly mutations that would affect other traits or that trade-offs have already been resolved through fixed compensatory mutations. The negative pleiotropic effects associated with resistance mutations can be masked by compensatory mutations occurring in the genetic background (Becher and Wirsel 2012; Cools et al. 2013). Fitness costs could also manifest for different traits than we analyzed (e.g., virulence and competitive ability) or depend on specific environmental conditions that we did not consider.

Our study demonstrates how GWAS of a global collection of pathogen strains can recapitulate the emergence of fungicide resistance. The distinctive complements of resistance mutations found among populations reflect how the evolutionary trajectory of fungicide adaptation is complex and difficult to predict. The apparent lack of trade-offs to adapt to azole fungicides in $P$. nodorum highlights how more sustainable crop protection strategies are needed. An absence of trade-offs will contribute to a rapid decline in fungicide effectiveness and more widespread losses in crop production.

\section{Acknowledgments}

We thank Marcello Zala for assistance in the laboratory and Lea Stauber for helpful comments on a previous version of this manuscript. The Genetic Diversity Center (GDC) of ETH Zurich and the Functional Genomics Center in Zurich provided sequencing facilities. This study was financed in part by the Coordenação de Aperfeiçoamento de Pessoal de Nível Superior-Brasil (CAPES)—Finance Code 001.

\section{Supplementary Material}

Supplementary data are available at Genome Biology and Evolution online.

\section{Literature Cited}

Abdolrasouli A, et al. 2015. Genomic context of azole resistance mutations in Aspergillus fumigatus determined using whole-genome sequencing Taylor, JW, editor. mBio 6(3):e00536.

Avenot HF, Michailides TJ. 2007. Resistance to Boscalid fungicide in Alternaria alternata isolates from pistachio in California. Plant Dis. 91(10):1345-1350.

Balfourier $F$, et al. 2019. Worldwide phylogeography and history of wheat genetic diversity. Sci Adv. 5(5):eaav0536.

Balter M. 2007. Seeking agriculture's ancient roots. Science 316(5833):1830-1835.

Barrett R, Schluter D. 2008. Adaptation from standing genetic variation. Trends Ecol Evol. 23(1):38-44. 
Bates D, Mächler M, Bolker B, Walker S. 2015. Fitting linear mixed-effects models using Ime4. J Stat Soft. 67(1), doi:10.18637/jss.v067.i01

Baucom RS. 2019. Evolutionary and ecological insights from herbicideresistant weeds: what have we learned about plant adaptation, and what is left to uncover? New Phytol. 223(1):68-82.

Becher R, Wirsel SGR. 2012. Fungal cytochrome P450 sterol 14 $\alpha$-demethylase (CYP51) and azole resistance in plant and human pathogens. Appl Microbiol Biotechnol. 95(4):825-840.

Bennett RS, Milgroom MG, Sainudiin R, Cunfer BM, Bergstrom GC. 2007. Relative contribution of seed-transmitted inoculum to foliar populations of Phaeosphaeria nodorum. Phytopathology 97(5):584-591.

Blixt E, Djurle A, Yuen J, Olson $\AA$. 2009. Fungicide sensitivity in Swedish isolates of Phaeosphaeria nodorum. Plant Pathol. 58(4):655-664.

Blixt E, Olson Å, Lindahl B, Djurle A, Yuen J. 2010. Spatiotemporal variation in the fungal community associated with wheat leaves showing symptoms similar to stagonospora nodorum blotch. Eur J Plant Pathol. 126(3):373-386.

Bolger A, Lohse M, Usadel B. 2014. Trimmomatic: a flexible trimmer for Illumina sequence data. Bioinformatics 30(15):2114-2120.

Bradbury P, et al. 2007. TASSEL: software for association mapping of complex traits in diverse samples. Bioinformatics 23(19):2633-2635.

Brunner PC, Stefansson TS, Fountaine J, Richina V, McDonald BA. 2016. A global analysis of CYP51 diversity and azole sensitivity in Rhynchosporium commune. Phytopathology 106(4):355-361.

Campbell GF, Crous PW. 2002. Fungicide sensitivity of South African netand spot-type isolates of Pyrenophora teres to ergosterol biosynthesis inhibitors. Austral Plant Pathol. 31(2):151.

Cheung HW. 2004. Id-1-induced Raf/MEK pathway activation is essential for its protective role against taxol-induced apoptosis in nasopharyngeal carcinoma cells. Carcinogenesis 25(6):881-887.

Chewapreecha C, et al. 2014. Comprehensive identification of single nucleotide polymorphisms associated with beta-lactam resistance within pneumococcal mosaic genes Guttman, DS, editor. PLoS Genet. 10(8):e1004547.

Ciudad T, Hickman M, Bellido A, Berman J, Larriba G. 2016. Phenotypic consequences of a spontaneous loss of heterozygosity in a common laboratory strain of Candida albicans. Genetics 203(3):1161-1176.

Cools HJ, Fraaije BA. 2013. Update on mechanisms of azole resistance in Mycosphaerella graminicola and implications for future control. Pest Manag Sci. 69(2):150-155.

Cools HJ, Hawkins N, Fraaije B. 2013. Constraints on the evolution of azole resistance in plant pathogenic fungi. Plant Pathol. 62:36-42.

Cools HJ, et al. 2010. Heterologous expression of mutated eburicol 14demethylase (CYP51) proteins of Mycosphaerella graminicola to assess effects on azole fungicide sensitivity and intrinsic protein function. AEM 76(9):2866-2872.

Coste $A$, et al. 2006. A mutation in Tac1p, a transcription factor regulating $C D R 1$ and CDR2, is coupled with loss of heterozygosity at chromosome 5 to mediate antifungal resistance in Candida albicans. Genetics 172(4):2139-2156.

Croll D, McDonald BA. 2017. The genetic basis of local adaptation for pathogenic fungi in agricultural ecosystems. Mol Ecol. 26(7):2027-2040.

Danecek $P$, et al. 2011. The variant call format and VCFtools. Bioinformatics 27(15):2156-2158.

Deng J, Carbone I, Dean RA. 2007. The evolutionary history of cytochrome P450 genes in four filamentous Ascomycetes. BMC Evol Biol. 7(1):30.

De Rossi $E$, et al. 2002. The multidrug transporters belonging to major facilitator superfamily (MFS) in Mycobacterium tuberculosis. Mol Med. 8(11):714-724

Dettman JR, Rodrigue N, Schoustra SE, Kassen R. 2017. Genomics of compensatory adaptation in experimental populations of Aspergillus nidulans. G3 7:427-436 de Waard MA, et al. 2006. Impact of fungal drug transporters on fungicide sensitivity, multidrug resistance and virulence. Pest Manag Sci. 62:195-207.

Downie RC, et al. 2018. Assessing European wheat sensitivities to Parastagonospora nodorum necrotrophic effectors and finemapping the Snn3-b1 locus conferring sensitivity to the effector SnTox3. Front Plant Sci. 9:881.

Dunkel N, Blaß J, Rogers PD, Morschhäuser J. 2008. Mutations in the multi-drug resistance regulator MRR1, followed by loss of heterozygosity, are the main cause of MDR1 overexpression in fluconazoleresistant Candida albicans strains. Mol Microbiol. 69(4):827-840.

Estep LK, et al. 2015. Emergence and early evolution of fungicide resistance in North American populations of Zymoseptoria tritici. Plant Pathol. 64(4):961-971.

Evanno G, Regnaut S, Goudet J. 2005. Detecting the number of clusters of individuals using the software structure: a simulation study. Mol Ecol. 14(8):2611-2620.

Farhat MR, et al. 2019. GWAS for quantitative resistance phenotypes in Mycobacterium tuberculosis reveals resistance genes and regulatory regions. Nat Commun. 10(1):2128.

Ficke A, Cowger C, Bergstrom GC, Brodal G. 2018. Understanding yield loss and pathogen biology to improve disease management: Septoria nodorum blotch-a case study in wheat. Plant Dis. 102(4):696-707.

Finn RD, Clements J, Eddy SR. 2011. HMMER web server: interactive sequence similarity searching. Nucleic Acids Res. 39(suppl):W29-37.

Fisher MC, Hawkins NJ, Sanglard D, Gurr SJ. 2018. Worldwide emergence of resistance to antifungal drugs challenges human health and food security. Science 360(6390):739-742.

Fisher MC, et al. 2012. Emerging fungal threats to animal, plant and ecosystem health. Nature 484(7393):186-194.

Fones H, Gurr S. 2015. The impact of Septoria tritici Blotch disease on wheat: an EU perspective. Fungal Genet Biol. 79:3-7.

Ford CB, et al. 2015. The evolution of drug resistance in clinical isolates of Candida albicans. elife 4:e00662.

Francis RM. 2017. pophelper: an R package and web app to analyse and visualize population structure. Mol Ecol Resour. 17(1):27-32.

Georgopapadakou NH, Walsh TJ. 1996. Antifungal agents: chemotherapeutic targets and immunologic strategies. Antimicrob Agents Chemother. 40(2):279-291.

Gisi U, Hermann D, Ohl L, Steden C. 1997. Sensitivity profiles of Mycosphaerella graminicola and Phytophthora infestans populations to different classes of fungicides. Pestic Sci. 51(3):290-298.

Hane JK, et al. 2007. Dothideomycete-plant interactions illuminated by genome sequencing and EST analysis of the wheat pathogen Stagonospora nodorum. Plant Cell 19(11):3347-3368.

Henderson PJF, Maiden MCJ. 1990. Homologous sugar transport proteins in Escherichia coli and their relatives in both prokaryotes and eukaryotes. Philos Trans R Soc Lond B Biol Sci. 326(1236):391-410.

Heun M. 1997. Site of einkorn wheat domestication identified by DNA fingerprinting. Science 278(5341):1312-1314.

Hill JA, Ammar R, Torti D, Nislow C, Cowen LE. 2013. Genetic and genomic architecture of the evolution of resistance to antifungal drug combinations Fay, JC, editor. PLoS Genet. 9(4):e1003390.

Hirai T, Subramaniam S. 2004. Structure and transport mechanism of the bacterial oxalate transporter OxIT. Biophys J. 87(5):3600-3607.

Hobbelen PHF, Paveley ND, van den Bosch F. 2014. The emergence of resistance to fungicides Sturtevant, J, editor. PLoS One 9(3):e91910.

Holt KE, et al. 2015. Genomic analysis of diversity, population structure, virulence, and antimicrobial resistance in Klebsiella pneumoniae, an urgent threat to public health. Proc Natl Acad Sci U S A. 112(27):E3574-3581.

Ingvarsson PK. 2005. Nucleotide polymorphism and linkage disequilibrium within and among natural populations of European aspen (Populus tremula L., Salicaceae). Genetics 169(2):945-953. 
Karaoglanidis GS, Thanassoulopoulos CC, loannidis PM. 2001. Fitness of Cercospora beticola field isolates — resistant and — sensitive to demethylation inhibitor fungicides. Eur J Plant Pathol. 107(3):337-347.

Keller S, McDermott J, Pettway R, Wolfe M, McDonald B. 1997. Gene flow and sexual reproduction in the wheat glume blotch pathogen Phaeosphaeria nodorum (anamorph Stagonospora nodorum). Phytopathology 87(3):353-358.

Knorr K, Jørgensen LN, Nicolaisen M. 2019. Fungicides have complex effects on the wheat phyllosphere mycobiome. PLoS One 14(3): $\mathrm{e} 0213176$

Kraiczy P, et al. 1996. The molecular basis for the natural resistance of the cytochrome bc1 complex from strobilurin-producing basidiomycetes to center QP inhibitors. Eur J Biochem. 235(1-2):54-63.

Kretschmer $\mathrm{M}$, et al. 2009. Fungicide-driven evolution and molecular basis of multidrug resistance in field populations of the grey mould fungus Botrytis cinerea. PLoS Pathog. 5(12):e1000696.

Lang Gl, Murray AW, Botstein D. 2009. The cost of gene expression underlies a fitness trade-off in yeast. Proc Natl Acad Sci U S A. 106(14):5755-5760

Langmead B, Salzberg S. 2012. Fast gapped-read alignment with Bowtie 2. Nat Methods 9(4):357-359.

Lass-Flörl C. 2011. Triazole antifungal agents in invasive fungal infections. Drugs 71(18):2405-2419.

Lendenmann MH, Croll D, McDonald BA. 2015. QTL mapping of fungicide sensitivity reveals novel genes and pleiotropy with melanization in the pathogen Zymoseptoria tritici. Fungal Genet Biol. 80:53-67.

Lendenmann MH, Croll D, Stewart EL, McDonald BA. 2014. Quantitative trait locus mapping of melanization in the plant pathogenic fungus Zymoseptoria tritici. G3 4:2519-2533.

Leslie T, Baucom RS. 2014. De novo assembly and annotation of the transcriptome of the agricultural weed Ipomoea purpurea uncovers gene expression changes associated with herbicide resistance. G3 4:2035-2047.

Levin BR, Perrot V, Walker N. 2000. Compensatory mutations, antibiotic resistance and the population genetics of adaptive evolution in bacteria. Genetics 154(3):985-997.

$\mathrm{Li} \mathrm{H}$, et al. 2009. The sequence alignment/map format and SAMtools. Bioinformatics 25(16):2078-2079.

Liu J, et al. 2015. A novel sterol regulatory element-binding protein gene (sreA) identified in Penicillium digitatum is required for prochloraz resistance, full virulence and erg11 (CYP51) regulation Cramer, RA, editor. PLoS One 10(2):e0117115.

Liu X, et al. 2011. Paralogous CYP51 genes in Fusarium graminearum mediate differential sensitivity to sterol demethylation inhibitors. Fungal Genet Biol. 48(2):113-123.

Lucas JA, Hawkins NJ, Fraaije BA. 2015. The evolution of fungicide resistance. Adv Appl Microbiol. 90:29-92.

Magrane M, Consortium U. 2011. UniProt knowledgebase: a hub of integrated protein data. Database 2011(0):bar009.

Manolio TA. 2013. Bringing genome-wide association findings into clinical use. Nat Rev Genet. 14(8):549-558.

Marchler-Bauer A, et al. 2015. CDD: NCBI's conserved domain database. Nucleic Acids Res. 43(D1):D222-226.

Massari ME, Murre C. 2000. Helix-loop-helix proteins: regulators of transcription in eucaryotic organisms. Mol Cell Biol. 20(2):429-440.

McDonald BA. 2015. How can research on pathogen population biology suggest disease management strategies? The example of barley scald (Rhynchosporium commune). Plant Pathol. 64(5):1005-1013.

McDonald BA, Linde C. 2002. Pathogen population genetics, evolutionary potential, and durable resistance. Annu Rev Phytopathol. 40(1):349-379.

McDonald MC, Oliver RP, Friesen TL, Brunner PC, McDonald BA. 2013. Global diversity and distribution of three necrotrophic effectors in
Phaeosphaeria nodorum and related species. New Phytol. 199(1):241-251.

McDonald MC, Razavi M, Friesen TL, Brunner PC, McDonald BA. 2012. Phylogenetic and population genetic analyses of Phaeosphaeria nodorum and its close relatives indicate cryptic species and an origin in the Fertile Crescent. Fungal Genet Biol. 49(11):882-895.

McDonald MC, et al. 2019. Rapid parallel evolution of azole fungicide resistance in Australian populations of the wheat pathogen Zymoseptoria tritici. Appl Environ Microbiol. 85:e01908.

McKenna A, et al. 2010. The genome analysis toolkit: a MapReduce framework for analyzing next-generation DNA sequencing data. Genome Res. 20(9):1297-1303.

Mikaberidze A, McDonald BA. 2015. Fitness cost of resistance: impact on management. In: Ishii $\mathrm{H}$, Hollomon DW, editors. Fungicide resistance in plant pathogens: principles and a guide to practical management. Tokyo (Japan): Springer. p. 77-89.

Mohd-Assaad N, McDonald BA, Croll D. 2016. Multilocus resistance evolution to azole fungicides in fungal plant pathogen populations. Mol Ecol. 25(24):6124-6142.

Moura de Sousa J, Balbontín R, Durão P, Gordo I. 2017. Multidrug-resistant bacteria compensate for the epistasis between resistances De Visser, A, editor. PLoS Biol. 15(4):e2001741.

Mullis MN, Matsui T, Schell R, Foree R, Ehrenreich IM. 2018. The complex underpinnings of genetic background effects. Nat Commun. 9(1):3548.

Nakagawa S, Schielzeth H. 2013. A general and simple method for obtaining $R^{2}$ from generalized linear mixed-effects models $\mathrm{O}^{\prime} \mathrm{Hara}, \mathrm{RB}$, editor. Methods Ecol Evol. 4(2):133-142.

Nosanchuk JD, Casadevall A. 2006. Impact of melanin on microbial virulence and clinical resistance to antimicrobial compounds. Antimicrob Agents Chemother. 50(11):3519-3528.

Oliver R, Friesen T, Faris J, Solomon P. 2012. Stagonospora nodorum: from pathology to genomics and host resistance. Annu Rev Phytopathol. 50(1):23-43.

Omrane S, et al. 2015. Fungicide efflux and the MgMFS1 transporter contribute to the multidrug resistance phenotype in Zymoseptoria tritici field isolates. Environ Microbiol. 17(8):2805-2823.

Omrane S, et al. 2017. Plasticity of the MFS1 promoter leads to multidrug resistance in the wheat pathogen Zymoseptoria tritici. mSphere 2(5):e00393.

Pagnout C, et al. 2019. Pleiotropic effects of rfa-gene mutations on Escherichia coli envelope properties. Sci Rep. 9(1):9696.

Pasrija R, Banerjee D, Prasad R. 2007. Structure and function analysis of CaMdr1p, a major facilitator superfamily antifungal efflux transporter protein of Candida albicans: identification of amino acid residues critical for drug/ $\mathrm{H}^{+}$transport. Eukaryotic Cell 6(3):443-453.

Paulsen IT, Brown MH, Skurray RA. 1996. Proton-dependent multidrug efflux systems. Microbiol Rev. 60(4):575-608.

Peng Y, et al. 2010. Characterization of the horseweed (Conyza canadensis) transcriptome using GS-FLX 454 pyrosequencing and its application for expression analysis of candidate non-target herbicide resistance genes. Pest Manag Sci. 66(10):1053-1062.

Pereira DA, Croll D, Brunner PC, McDonald BA. 2020. Natural selection drives population divergence for local adaptation in a wheat pathogen. Fungal Genet Biol. 141:103398.

Pereira DA, McDonald BA, Brunner PC. 2017. Mutations in the CYP51 gene reduce DMI sensitivity in Parastagonospora nodorum populations in Europe and China. Pest Manag Sci. 73(7):1503-1510.

Power RA, Parkhill J, de Oliveira T. 2017. Microbial genome-wide association studies: lessons from human GWAS. Nat Rev Genet. 18(1):41-50.

Powles SB, Yu Q. 2010. Evolution in action: plants resistant to herbicides. Annu Rev Plant Biol. 61(1):317-347. 
Pritchard JK, Stephens M, Donnelly P. 2000. Inference of population structure using multilocus genotype data. Genetics 155(2):945-959.

Quevillon E, et al. 2005. InterProScan: protein domains identifier. Nucleic Acids Res. 33(Web Server):W116-120.

Quinlan AR, Hall IM. 2010. BEDTools: a flexible suite of utilities for comparing genomic features. Bioinformatics 26(6):841-842.

Redhu AK, Shah AH, Prasad R. 2016. MFS transporters of Candida species and their role in clinical drug resistance. FEMS Yeast Res. 16(4):fow043.

Richards JK, Wyatt NA, Liu Z, Faris JD, Friesen TL. 2017. Reference quality genome assemblies of three Parastagonospora nodorum isolates differing in virulence on wheat. G3 8:93.300462.2017.

Richards JK, et al. 2019. Local adaptation drives the diversification of effectors in the fungal wheat pathogen Parastagonospora nodorum in the United States. PLoS Genet. 15(10):e1008223.

Ritz C, Baty F, Streibig J, Gerhard D. 2015. Dose-response analysis using R. PLoS One 10(12):e0146021.

Rosas ÁL, et al. 2000. Synthesis of polymerized melanin by Cryptococcus neoformans in infected rodents Kozel, TR, editor. Infect Immun. 68(5):2845-2853.

Schenk MF, de Visser JAG. 2013. Predicting the evolution of antibiotic resistance. BMC Biol. 11(1):14.

Schneider CA, Rasband WS, Eliceiri KW. 2012. NIH image to ImageJ: 25 years of image analysis. Nat Methods 9(7):671-675.

Schwarz G. 1978. Estimating the dimension of a model. Ann Stat. 6(2):461-464.

Selmecki A, Forche A, Berman J. 2010. Genomic plasticity of the human fungal pathogen Candida albicans. Eukaryot Cell 9(7):991-1008.

Shin J-H, Blay S, Graham J, McNeney B. 2006. LDheatmap: an $R$ function for graphical display of pairwise linkage disequilibria between single nucleotide polymorphisms. J Stat Soft. 16(Code Snippet 3), doi:10.18637/jss.v016.c03

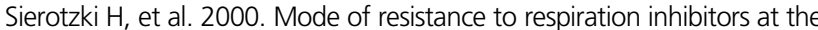
cytochrome bc1 enzyme complex of Mycosphaerella jiensis field isolates. Pest Manag Sci. 56(10):833-841.

Slater G, Birney E. 2005. Automated generation of heuristics for biological sequence comparison. BMC Bioinformatics 6(1):31

Solomon PS, Lowe RG, Tan K-C, Waters OD, Oliver RP. 2006 Stagonospora nodorum: cause of stagonospora nodorum blotch of wheat. Mol Plant Pathol. 7(3):147-156.

Sommerhalder RJ, McDonald BA, Zhan J. 2006. The Frequencies and spatial distribution of mating types in Stagonospora nodorum are consistent with recurring sexual reproduction. Phytopathology 96(3):234-239.

Stehmann C, de Waard MA. 1996. Sensitivity of populations of Botrytis cinerea to triazoles, benomyl and vinclozolin. Eur J Plant Pathol. 102(2):171-180.

Stergiopoulos I, Zwiers L-H, de Waard MA. 2002 Secretion of natural and synthetic toxic compounds from filamentous fungi by membrane transporters of the ATP-binding cassette and major facilitator superfamily. In: Logrieco A, Bailey JA, Corazza L, Cooke BM, editors. Mycotoxins in plant disease. Dordrecht (The Netherlands): Springer. p. 719-734.

Storey J, Tibshirani R. 2003. Statistical significance for genome wide studies. Proc Natl Acad Sci U S A. 100(16):9440-9445.
Strange RN, Scott PR. 2005. Plant disease: a threat to global food security. Annu Rev Phytopathol. 43(1):83-116.

Stukenbrock EH, Banke S, McDonald BA. 2006. Global migration patterns in the fungal wheat pathogen Phaeosphaeria nodorum. Mol Ecol. 15(10):2895-2904

Stukenbrock EH, McDonald BA. 2008. The origins of plant pathogens in agro-ecosystems. Annu Rev Phytopathol. 46(1):75-100.

Syme RA, et al. 2018. Pan-Parastagonospora comparative genome analysis - effector prediction and genome evolution. Genome Biol Evol. 10(9):2443-2457.

Tang Y, et al. 2016. GAPIT version 2: an enhanced integrated tool for genomic association and prediction. Plant Gen. 9:1-9.

Torriani SF, Brunner PC, McDonald BA, Sierotzki H. 2009. Qol resistance emerged independently at least 4 times in European populations of Mycosphaerella graminicola. Pest Manag Sci. 65(2):155-162.

Tranel PJ, Wright TR. 2002. Resistance of weeds to ALS-inhibiting herbicides: what have we learned? Weed Sci. 50(6):700-712.

Trindade S, et al. 2009. Positive epistasis drives the acquisition of multidrug resistance Zhang, J, editor. PLoS Genet. 5(7):e1000578.

van den Bosch, F Gilligan, CA. 2008. Models of fungicide resistance dynamics. Annu Rev Phytopathol. 46(1):123-147.

van den Bosch F, Paveley N, Shaw M, Hobbelen P, Oliver R. 2011. The dose rate debate: does the risk of fungicide resistance increase or decrease with dose? The dose rate debate. Plant Pathol. 60(4):597-606.

Vandeputte DAA, et al. 2002. Expression and distribution of id helix-loophelix proteins in human astrocytic tumors. Glia 38(4):329-338.

VanRaden PM. 2008. Efficient methods to compute genomic predictions. J Dairy Sci. 91(11):4414-4423.

Wellings CR. 2007. Puccinia striiformis in Australia: a review of the incursion, evolution, and adaptation of stripe rust in the period 1979-2006. Aust J Agric Res. 58(6):567.

Wyand RA, Brown JKM. 2005. Sequence variation in the CYP51 gene of Blumeria graminis associated with resistance to sterol demethylase inhibiting fungicides. Fungal Genet Biol. 42(8):726-735.

Yamashita M, Fraaije B. 2018. Non-target site SDHI resistance is present as standing genetic variation in field populations of Zymoseptoria tritici: non-target site SDHI resistance in Zymoseptoria tritici. Pest Manag Sci. 74(3):672-681.

Yan $X$, et al. 2011. A sterol $14 \alpha$-demethylase is required for conidiation, virulence and for mediating sensitivity to sterol demethylation inhibitors by the rice blast fungus Magnaporthe oryzae. Fungal Genet Biol. 48(2):144-153.

Yang C, Hamel C, Vujanovic V, Gan Y. 2011. Fungicide: modes of action and possible impact on nontarget microorganisms. ISRN Ecol. 2011:1-8

Yu J, et al. 2006. A unified mixed-model method for association mapping that accounts for multiple levels of relatedness. Nat Genet. 38(2):203-208.

Zhan J, McDonald BA. 2013. Field-based experimental evolution of three cereal pathogens using a mark-release-recapture strategy. Plant Pathol. 62:106-114.

Associate editor: Li-Jun Ma 